\title{
Cystic Nephroma with Pleuropulmonary Blastoma: A Rare Dual Pathology
}

\author{
Kiran Hilal, Anam Khan, Nida Sajjad, Kumail Khandwala*
}

Department of Radiology, Aga Khan University Hospital, Karachi, Pakistan.

\begin{abstract}
Cystic nephroma $(\mathrm{CN})$ belongs to a heterogeneous group of renal cystic tumors while pleuropulmonary blastoma is an uncommon pediatric lung neoplasm, believed to be originating either from lung tissue itself or from the pleura. Synchronous development of pleuropulmonary blastoma following cystic nephroma is a rare entity in pediatric patients. The etiology and pathogenesis of this dual pathology is still unknown. We report a case of a child who presented with an abdominal mass diagnosed as cystic nephroma on surgical resection, who later developed a thoracic lesion which was subsequently diagnosed as pleuropulmonary blastoma.
\end{abstract}

Key words: Cystic nephroma; Pleuropulmonary blastoma; Renal cystic tumor

Correspondence*: Kumail Khandwala, Department of Radiology, Aga Khan University Hospital, Karachi, Pakistan.

E-mail: kumail.khandwala@gmail.com @ 2018, Hilal et al.

Submitted: 05-10-2017 Accepted: 03-12-2017

Conflict of Interest: None Source of Support: Nil

This is an open-access article distributed under the terms of the Creative Commons Attribution License, which permits unrestricted use, distribution, and reproduction in any medium, provided the original work is properly cited.

\section{CASE REPORT}

A 12-month old child presented with the complaints of fever, abdominal pain and abdominal distension for three months. On examination a firm, non-tender mass was palpable in the left upper abdomen. CT scan of abdomen revealed a well-defined, multiloculated predominantly cystic mass lesion arising from interpolar region of left kidney compressing the normal renal parenchyma posterolaterally (Fig. $1 \mathrm{~A}$ ).

Provisional diagnosis of a neoplastic renal lesion was made, with features favoring cystic Wilms' tumor. Subsequently, left radical nephrectomy was performed and an encapsulated mass measuring approximately $9 \times 10 \times 10 \mathrm{~cm}$ was excised which on cut section exhibited multiple cystic areas filled with serous fluid. Histopathology of the specimen showed renal parenchyma exhibiting a neoplastic lesion comprising of variable sized cystic dilated spaces lined by flattened, cuboidal and hobnail epithelial lining. The intervening stroma was fibrocellular and the capsule was intact. The ureteric resection margin was histologically unremarkable. These features were consistent with multicystic nephroma. Patient remained stable postoperatively. On follow up CT scan done two months later, there was no evidence of residual disease (Fig.1B).

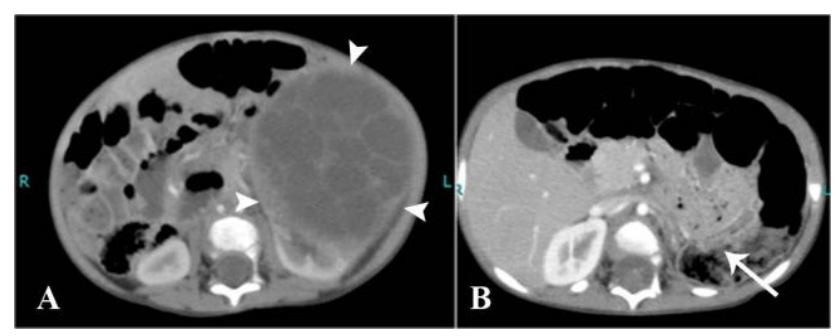

FIGURE 1 A: CT abdomen axial section shows well-defined predominantly cystic multiloculated mass lesion arising from left kidney (arrowheads), displacing the surrounding structures and aorta. B: Follow up CT abdomen shows removal of mass and left nephrectomy with no evidence of residual disease (white arrow).

One year later, the patient presented with complaints of cough and respiratory distress for two weeks. On ex- 
amination decreased air entry and dull percussion was noted over the left lung field. Chest x-ray showed masslike homogeneous opacification of left upper lobe which was causing contralateral mediastinal shift. CT chest was performed which showed a large well-defined heterogeneously enhancing mass involving the left upper thoracic cavity, measuring approximately $8 \times 6 \mathrm{~cm}$. This lesion was resulting in displacement of heart and aortic arch towards the right side and also resulting in compression of the pulmonary artery and veins of the left upper lobe (Fig.2).

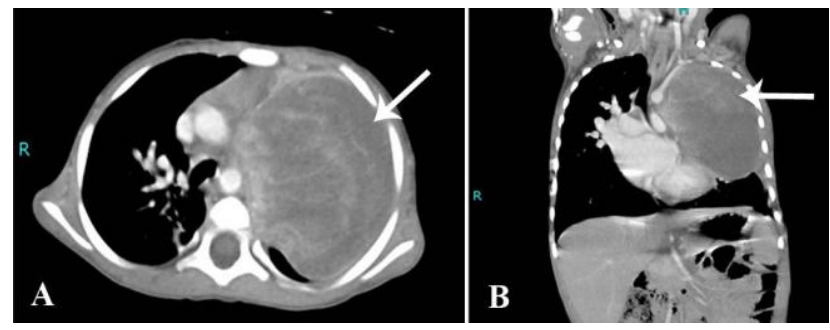

FIGURE 2 A \& B: CT chest post contrast axial and coronal images showing a large heterogeneous mass involving the left upper thoracic cavity (white arrows) causing significant mass effect over mediastinal vessels and heart.

Renal bed on the left side appeared clear without any residual disease. Cardiothoracic surgery team was involved. Excision of the thoracic mass was performed with marginal excision of left upper lobe of lung. Histopathology of the excised specimen revealed an infiltrating neoplastic lesion arranged in diffuse sheets and nodules. Neoplastic cells were markedly pleomorphic and had bi-lobed to multi lobed vesicular nuclei with variably prominent nucleoli along with moderate to scant cytoplasm. Prominent mitotic activity was seen along with atypical mitosis. Neoplastic cells were set in a fibromyxoid background. Areas of necrosis and pseudorosette formation were also seen. Pleural surface showed desmoplastic response. These neoplastic cells were positive from immunohistochemical Vimentin, Desmin and CD99(Mic-2). Immunohistochemical CKAE1/AE3 was negative. The features were consistent with pleuropulmonary blastoma type III. The tumor was present at a distance of $0.3 \mathrm{~cm}$ from the pleural surface. The left upper lobe bronchial resection margin was involved by the tumor. The patient was initially kept under special care and subsequently extubated. Chest physiotherapy and rehabilitation was initiated and he was discharged after stabilization.

Few weeks later a follow up CT scan was performed which showed interval development of multiple low attenuation pleural based lesions adjacent to the left low- er lobe predominantly along the posterior chest wall, representing disease recurrence. No evidence of any recurrent disease was identified in the left renal bed.

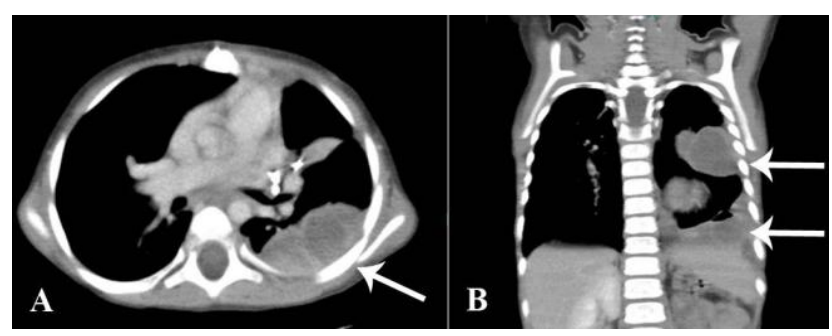

FIGURE 3 A \& B: Post surgical follow-up CT chest after removal of left thoracic mass. Axial and coronal images showing interval development of multiple new pleural based lesions predominately along the posterior chest wall representing recurrence (white arrows).

The patient was readmitted with complaints of respiratory difficulty. Treatment option of chemotherapy was provided, however due to poor prognosis it was refused by patient's parents. The patient is now under palliative care with "do not resuscitate" status.

\section{DISCUSSION}

Cystic nephroma is a pediatric renal neoplasm. It is commonly unilateral however may rarely present bilaterally. On CT scan the appearance is typically of a wellencapsulated mass with multiple non-communicating fluid filled cystic spaces. [1-4] It usually follows a benign course and nephrectomy is curative, however rarely local recurrence may occur which is effectively treated by radiotherapy or chemotherapy. [3] Histopathologic characteristic of multicystic renal mass cannot be predicted by its clinical and imaging features, thus surgery remains essential for both the diagnosis and treatment. [6] Although $\mathrm{CN}$ is a benign tumor, patients with a past history of cystic nephroma need to be closely followedup for development of this and other forms of neoplasia. Having said this, active surveillance could unfortunately not be done in our case as the family belonged to a rural background and had lost to follow-up after the first surgery.

Pleuropulmonary blastoma (PPB), on the other hand, is a rare and aggressive pediatric lung neoplasm. Clinical presentation of PPB usually mimics common respiratory problems and is often mistaken for pneumonia leading to a delay in diagnosis. The tumor usually originates from the lung tissue itself and is located at the periphery of the lung, however it may be extrapulmonary involving the mediastinum, diaphragm or pleura, which signifies a poor prognosis. Synchronous and metachronous tumors are not uncommon in patients of pleuropulmonary 
AJS CR

blastoma. Metastasis is commonly to brain, bone, lymph nodes, liver, pancreas, kidney and adrenals. [7-9] The sections from the chest in the initial CT scan did not reveal any lesion in the thorax, therefore suggesting a metachronous development of pleuropulmonary blastoma. Histologically, it is characterized by primitive blastema with malignant mesenchymal stroma showing multidirectional differentiation. According to classification by Dehner et al, three types of pleuropulmonary blastoma are recognized depending upon its cystic and solid component. Type $I$ is purely cystic without any macroscopic solid component. This form provides better survival and effective cure as compared to the other two forms. Type II contains both solid and cystic component while Type III is purely solid cancerous tumor. Pleuropulmonary blastoma is treated by surgery, chemotherapy and in some cases by radiotherapy.[7] Despite multimodal therapeutic approach, pleuropulmonary blastoma has a poor prognosis.

The association of PPB and CN is noteworthy. This pathology appears to have a genetic basis; however the specific association is not yet fully established. Metachronous association has not been described previously. According to Kousari et al, a familial syndrome called DICER1-PPB is found in $10 \%$ of patients with pleuropulmonary blastoma or in their family members. This mutation in the DICER1 gene appears to have a strong relation with the development of cystic nephroma in these patients with PPB. Therefore, they recommend regular abdominal screening of patients with this familial variant of PPB.[10] The latent time period between the development of both tumors was assumed to be one year in our patient. The developmental nature of these neoplasms suggests that the abnormality may have an important role linked to embryogenesis and organogenesis of the lung and kidney. Research to establish the origin, diagnostic procedures and management protocol are ongoing, however due to the rarity of this pathology case collection from multiple hospitals is required to determine the exact association.
Cystic Nephroma with Pleuropulmonary Blastoma: A Rare Dual Pathology

Consent: Authors have submitted signed consent form from legal guardian of the patient and available with editorial office.

Authors' Contribution: All authors were involved in manuscript writing, literature review, and final approval of the manuscript.

\section{REFERENCES}

1. Bagley JE, Berry JL, McMurrian K. Case report: Multimodality imaging of a cystic nephroma. Radiol Technol. 2013; 84:559-66.

2. Sodhi KS, Suri S, Samujh R, Rao KL, Vaiphei K, Saxena AK. Case report: bilateral multilocular cystic nephromas: a rare occurrence. Br J Radiol. 2005; 78:450-2.

3. Bhardwaj AK, Sharma PD, Mittal A, Sharma A. Bilateral cystic nephroma with pleuropulmonary blastoma. BMJ Case Rep. 2011.

4. Madewell JE, Goldman SM, Davis CJ, Jr., Hartman DS, Feigin DS, Lichtenstein JE. Multilocular cystic nephroma: a radiographic-pathologic correlation of 58 patients. Radiology. 1983; 146:309-21.

5. Priest JR, McDermott MB, Bhatia S, Watterson J, Manivel JC, Dehner LP. Pleuropulmonary blastoma: a clinicopathologic study of 50 cases. Cancer. 1997; 80:14761.

6. Agrons GA, Wagner BJ, Davidson AJ, Suarez ES. Multilocular cystic renal tumor in children: radiologicpathologic correlation. Radiographics. 1995; 15:653-69.

7. Agarwal AK, Das S, Agarwal A, Ghosh D, Chaterjee N, Pal MS. Pleuropulmonary blastema with cystic nephroma - A rare presentation and surgical dilemma. Indian $\mathrm{J}$ Pediatr. 2008; 75:1266-8.

8. Dehner LP, Watterson J, Priest JR. Pleuropulmonary blastoma. A unique intrathoracic-pulmonary neoplasm of childhood. Perspect Pediatr Pathol. 1995; 18:214-26.

9. Yusuf U, Dufour D, Jenrette JM, 3rd, Abboud MR, Laver J, Barredo JC. Survival with combined modality therapy after intracerebral recurrence of pleuropulmonary blastoma. Med Pediatr Oncol. 1998; 30:63-6.

10. Kousari YM, Khanna G, Hill DA, Dehner LP. Case 211: pleuropulmonary blastoma in association with cystic nephroma-DICER1 syndrome. Radiology. 2014; 273:6225. 